\title{
Big Bang Nucleosynthesis with Bound States of Long-lived Charged Particles
}

\author{
Manoj Kaplinghat and Arvind Rajaraman \\ Department of Physics and Astronomy \\ University of California, Irvine, California 92697, USA
}

(Dated: October 7, 2018)

\begin{abstract}
Charged particles $(\mathrm{X})$ decaying after primordial nucleosynthesis are constrained by the requirement that their decay products should not change the light element abundances drastically. If the decaying particle is negatively charged $\left(\mathrm{X}^{-}\right)$then it will bind to the nuclei. We consider the effects of the decay of $\mathrm{X}$ when bound to Helium- 4 and show that this will modify the Lithium abundances.
\end{abstract}

\section{INTRODUCTION}

Many models of physics beyond the standard model have long-lived charged particles in their spectrum. For example, in the class of supergravity models where the gravitino is the lightest supersymmetric particle (LSP), the next lightest (NLSP) can be a long-lived charged slepton [1]. Similar phenomenology arises in Universal Extra Dimension models 22]. Another example is the class of SUSY models with axino dark matter where again the NLSP could be long-lived [3, 4, [5]. A third example is a supersymmetric model with almost degenerate LSP and NLSP such that the dominant decay channel for the NLSP is kinematically suppressed [6, 7].

Here we consider a new aspect of the early universe cosmology of these particles that affects the light element abundances. Other effects of these charged particles were previously considered in $[8,9,10,11,12,13,14,15,16$, $17,18,19,20,21$.

If the particle is negatively charged, it will eventually form a bound state with the positively charged nuclei. When the particle decays, the decay products will electromagnetically interact with the nearby nucleus. If the nucleus is ${ }^{4} \mathrm{He}$, it may break up, creating ${ }^{3} \mathrm{He}, \mathrm{T}, \mathrm{D}, \mathrm{p}$, n. These ${ }^{3} \mathrm{He}, \mathrm{T}$ and $\mathrm{D}$ nuclei will find other ${ }^{4} \mathrm{He}$ nuclei and interact to form ${ }^{6} \mathrm{Li}$ and ${ }^{7} \mathrm{Li}$.

In addition to the production mechanism described above, charged particle decays result in a large nonthermal photon background that can destroy the created ${ }^{6} \mathrm{Li}$ and ${ }^{7} \mathrm{Li}$ nuclei. When both the production and destruction processes are taken into account, we find that the overall ${ }^{6} \mathrm{Li}$ production could be in the same range as the Lithium isotopic abundance measured in few low metallicity popII stars 22, 23, 24, 25, 26, 27].

Other effects can come from the elastic scattering of the charged particle produced in the decay from the ${ }^{4} \mathrm{He}$ nucleus, and also from the change in the motion of the ${ }^{4} \mathrm{He}$ in the bound state. We find that these effects produce insignificant changes to standard BBN abundances.

In this work we only consider singly charged bound state of ${ }^{4}$ He nuclei [28, 29, 30, 31]. We ignore the production of neutral atoms of ${ }^{4} \mathrm{He}$ bound to two X. We also do not consider the bound state of protons. This binding process starts below about $0.5 \mathrm{keV}$ and could be important for lifetimes larger than about a year. Another interesting process we have not considered here is that of forming stable $\mathrm{Be}^{8}-\mathrm{X}$ bound states $\left[28\right.$ ]. A stable $\mathrm{Be}^{8}-\mathrm{X}$ bound state has important implications for the production of Carbon in the early universe. We hope to return to these issues in future work.

While this work was being completed, two related articles appeared on the arXiv. The three articles all consider different processes and hence complement each other. The first by Pospelov [32] pointed out that the nuclear processes with a photon in the final state will be modified by the presence of the strong electric field of the $\mathrm{X}$ particle. Pospelov showed that the enhanced D on $\mathrm{X}-{ }^{4} \mathrm{He}$ cross-section could produce orders of magnitude larger ${ }^{6} \mathrm{Li}$ abundance. However, it is important to note that if the decay lifetime is about $10^{6}$ seconds or larger, then ${ }^{6} \mathrm{Li}$ will also be destroyed due to the non-thermal photons from the decay.

The second paper by Kohri and Takayama [33] worked out the changes in the light element abundances due to the change in coulomb barrier in the presence of the $X^{-}$, and due to the changes in the kinematics and energy balance for the reactions. They found that the Lithium abundances can be significantly modified from the standard BBN predictions.

\section{RATES}

To calculate the magnitude of the effects we mentioned in the previous section, we need to calculate the following quantities.

1. $f_{b}$, the fraction of Helium-4 nuclei bound to the charged particles.

2. $f_{P}$, the fraction of the bound ${ }^{4} \mathrm{He}$ nuclei which are destroyed by photodisintegration.

3. $f_{3}$, the average fractions of ${ }^{3} \mathrm{He}$ and $\mathrm{T}$ produced by the photodisintegration of a ${ }^{4} \mathrm{He}$ nucleus.

4. $f_{L}$, the fraction of ${ }^{3} \mathrm{He}$ and $\mathrm{T}$ that formed in the photo-disintegration that find another ${ }^{4} \mathrm{He}$ nucleus and form ${ }^{6} \mathrm{Li}$. 


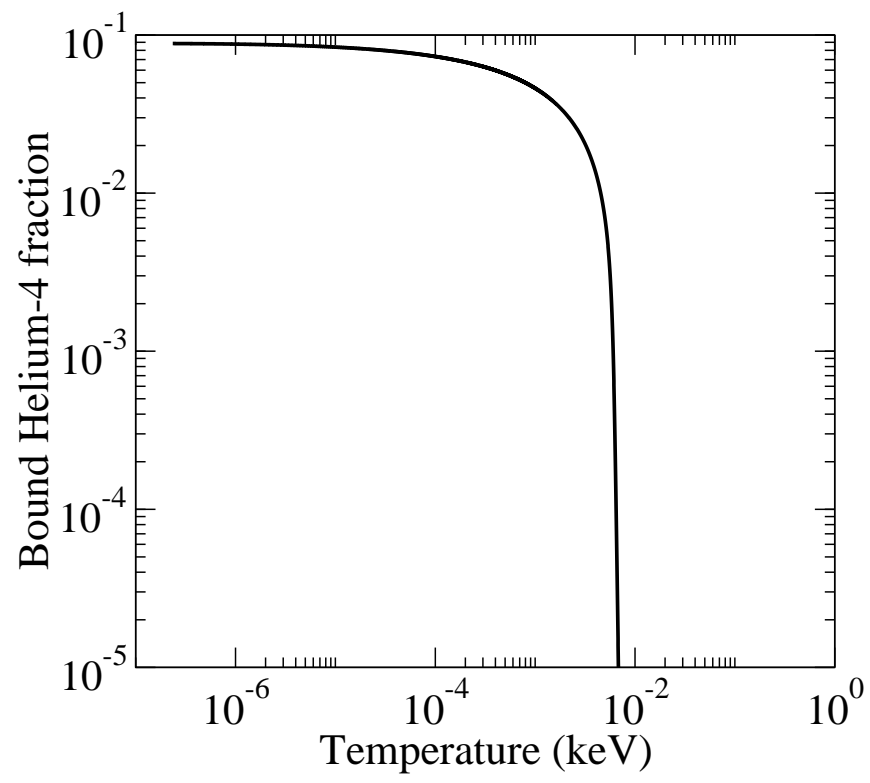

FIG. 1: Solid curve is the fraction of helium nuclei that are bound to a long-lived massive charged particle. The number density of these charged particles is set by the requirement that the massive neutral particle it decays into be all the dark matter in the universe and the curve here is for a dark matter particle mass of $200 \mathrm{GeV}$. We have taken the decay lifetime to be much larger than the age of the universe at the temperatures shown above.

In what follows we will concentrate on the ${ }^{6} \mathrm{Li}$ abundance. Some ${ }^{7} \mathrm{Li}$ is also produced through ${ }^{4} \mathrm{He}\left({ }^{3} \mathrm{He}, \gamma\right){ }^{7} \mathrm{Be}$ and ${ }^{4} \mathrm{He}(\mathrm{T}, \gamma){ }^{7} \mathrm{Li}$. However, these reactions have an electromagnetic component and hence are slower than the ${ }^{6} \mathrm{Li}$ production reactions.

The total fraction of ${ }^{6} \mathrm{Li}$ to Hydrogen (by number) is then $f_{b} f_{P} f_{3} f_{L} Y_{P} / 4 /\left(1-Y_{P} / 2\right)$ where $Y_{P}$ is the total ${ }^{4} \mathrm{He}$ mass fraction (bound or not) in the absence of the decay. The effects on the abundances of the other nuclei can also be similarly determined.

\section{CHARGED PARTICLE RECOMBINATION}

The bulk of ${ }^{4} \mathrm{He}$ forms at $T \equiv T_{\alpha, f} \simeq 70 \mathrm{keV}$. If the decay happens significantly before this temperature $T_{\alpha, f}$, then the effects we are interested in here are not important. For the rest of the discussion we will assume that the decay lifetime is larger than $1 / H\left(T_{\alpha, f}\right)$.

We also assume that the predominant component of $\mathrm{X}$ is negatively charged. Thus we are guaranteed that $\mathrm{X}$ will bind to the positively charged nuclei.

The equation for the evolution of the fraction of charged $\mathrm{X}$ particles that are not bound $\left(x_{f}\right)$ is given by

$$
\dot{x}_{f}=\left[\beta\left(1-x_{f}\right)-R x_{f} n_{B} \frac{1}{4} Y_{p}(t)\left(1-f_{b}\right)\right] .
$$

In the above equation, $R$ is the recombination rate and $\beta$ is the ionization rate. The recombination rate may be calculated in a manner analogous to the recombination of Hydrogen to yield $R=5(\alpha / m) \sqrt{(\mathrm{BE} / T)} \ln (\mathrm{BE} / T)$. The ionization cross-section and hence $\beta$ can then be found from the usual analysis of the equilibrium case.

\section{PHOTO-DISINTEGRATION OF HELIUM-4}

The photo-disintegration occurs through a one-photon exchange diagram, where the charged decay product emits a photon of invariant momentum $q^{2}=-Q^{2}$, which hits the Helium nucleus and breaks it up.

Photo-disintegration can occur as long as the energy transferred to the hadronic system, is larger than the binding energy, which is here $28.8 \mathrm{MeV}$. Unfortunately, the cross-section for the process $\gamma+H^{4} \rightarrow$ any has not been measured for all the energies that we need. For large $Q^{2}$, one can use the formulae for deep inelastic scattering, but these are only valid when $Q^{2}$ is greater than $Q_{0}^{2} \sim$ $1 G e V^{2}$.

To proceed, we will restrict our analysis to photon momenta satisfying $Q^{2}>Q_{0}^{2}$. This will result in a conservative estimate of the cross-section; the true cross-section will certainly receive additional contributions from photons below this cutoff.

For $Q^{2}>Q_{0}^{2}$, the cross section is given by

$$
\begin{aligned}
\frac{d \sigma_{D}}{d Q^{2}}= & \frac{2 \pi \alpha^{2}}{Q^{4}} \int_{0}^{1} d x \\
& \times \sum_{f} f_{f}(x) Q_{f}^{2}\left[1+\left(1-\frac{Q^{2}}{x s}\right)^{2}\right] \theta\left(x s-Q^{2}\right)
\end{aligned}
$$

Here $f_{f}(x)$ is the parton distribution function of flavor $f$ at longitudinal fraction $x . Q_{f}$ is the corresponding charge. The final factor above is a kinematic constraint.

Unlike the usual situation in deep inelastic scattering, here we should integrate over the allowed $Q^{2}$. For a fixed $x$ the range is $Q_{0}^{2}<Q^{2}<x s$. The allowed range of $x$ is then between $\frac{Q_{0}^{2}}{s}$ and 1 .

We then have to perform an integration over $x$. This is hampered by our lack of knowledge of $f_{f}(x)$. Qualitatively, $f_{f}(x)$ increases at smaller $x$, so this integral is expected to be dominated by the smaller values of $x$.

We will assume that $\epsilon=\frac{Q_{0}^{2}}{s} \ll 0.1$, and restrict the range of the integral between $x=\epsilon$ and $x=0.1$. Over this range, we can take approximately $F_{2}(x)=$ $x f_{f}(x) Q_{f}^{2} \sim 1$ [34].

With these approximations, we find

$$
\sigma \sim-\frac{2 \pi \alpha^{2}}{Q_{0}^{2}} \ln \left(10 Q_{0}^{2} / s\right)
$$

Using $1 \mathrm{GeV}^{-1} \sim 0.2 \mathrm{fm}$, the cross section evaluates to about $2 \pi \times 10^{-4} \times 0.04 \times 10^{-26} \mathrm{~cm}^{2} \ln \left(10 Q_{0}^{2} / \mathrm{s}\right) \sim$ 
$0.3 \times 10^{-3} \ln \left(10 Q_{0}^{2} / s\right) m b$ in almost exact agreement with a calculation from GEANT.

To derive a probability from this quantity, we must multiply by the flux, and for this we must examine the geometry. The helium nucleus orbits around $\mathrm{X}$ at a distance given by $d \simeq m^{-1} / Z \alpha=3.6 \mathrm{fm}$ where we have taken $m$ to be the mass of the helium nucleus. The size of the helium nucleus may be taken to be $r=1.44 A^{1 / 3} \mathrm{fm}=2.3 \mathrm{fm}$. The solid angle subtended by the nucleus at the position of $\mathrm{X}$ is $2 \pi(1-\cos \phi)$ where $\cos ^{2} \phi=1-r^{2} / d^{2}$ and the probability of disassociation is

$$
\frac{\sigma_{D}}{4 \pi d^{2}} \frac{2(1-\cos \phi)}{\sin ^{2} \phi \cos ^{2} \phi} \simeq 0.012 \sigma_{D} \mathrm{fm}^{-2} .
$$

The probability of an interaction between the emitted particle and the nucleus is thus $0.012 \times 3 \times 10^{-5}=3.6 \times$ $10^{-7}$ for a cross-section of $3 \times 10^{-4} \mathrm{mb}$.

\section{NUCLEI FROM THE PHOTO-DISINTEGRATION}

The photo-disintegration of ${ }^{4} \mathrm{He}$ described in the previous section typically breaks up the nucleus. The nuclei will split into lighter elements ${ }^{3} \mathrm{He}, \mathrm{T}, \mathrm{D}, \mathrm{p}$ and $\mathrm{n}$. The exclusive cross-sections for these processes are unknown. We will assume following 35] that the helium nucleus is always destroyed, and produces ${ }^{3} \mathrm{He}$ and $\mathrm{T}$ with the cross-sections $\sigma_{3}=\sigma_{D}=\frac{4}{9} \sigma_{P}$. In other words, the photodisintegration produces a ${ }^{3} \mathrm{He}$ or $\mathrm{T}$ nucleus in about half the interactions, and D in about half the interactions. We will assume that ${ }^{3} \mathrm{He}$ and $\mathrm{T}$ are produced in equal amounts.

\section{PRODUCTION OF LITHIUM-6}

The ${ }^{3} \mathrm{He}$ in the photo-dissociation of ${ }^{4} \mathrm{He}$ are extremely relativistic. They can then scatter off background ${ }^{4} \mathrm{He}$ nuclei, and produce Lithium. The rate for this is given by $\Gamma_{\alpha}=3.6 \times 10^{-15} Y_{P} T_{\mathrm{MeV}}^{3}(\sigma / 40 \mathrm{mb})\left(\eta_{10} / 6\right) \mathrm{MeV}$ where $\sigma$ is the appropriate nuclear cross-section. In comparison, the expansion rate of the universe is $H(T)=$ $2.5 \times 10^{-22} T_{\mathrm{MeV}}^{2} \mathrm{MeV}$. Comparing the two rates, we see that the nuclear reaction rate will be larger than the expansion rate of the universe until $T \sim 0.3 \mathrm{eV}(40 \mathrm{mb} /<$ $\sigma v>)$.

The nuclear reaction rate that we have quoted above assumes that the ${ }^{3} \mathrm{He}$ are energetic enough to overcome the Coulomb barrier. This is certainly true for the nuclei at the moment of decay. However, these nuclei will lose energy due to a variety of processes [9, 36], the most important of which are the energy losses due to Coulomb and Compton scattering.

The time scale for these energy loss processes is much shorter than the nuclear reaction rate as well as the ex- pansion rate. As a consequence one may write the probability of ${ }^{6} \mathrm{Li}$ to form as [8]

$$
f_{L}=\int_{E_{\mathrm{th}}}^{K_{0}} d K \sigma_{3+4}(K) v \frac{Y_{P}}{4} n_{B}(T)\left(\frac{d K}{d T}\right)^{-1}
$$

where $v$ is the relative speed, $K$ is the kinetic energy of the nucleon, $d K / d T$ is the total energy loss rate and $\sigma_{3+4}$ is the cross-section for ${ }^{3} \mathrm{He}$ on ${ }^{4} \mathrm{He}$ reaction. $E_{\mathrm{th}}$ is the minimum energy required for the reaction to occur. The upper limit to the kinetic energy $K_{0}$ is the energy with which the ${ }^{3} \mathrm{He}$ nucleus is born. As it turns out, the integral is insensitive to the upper limit as long as it is larger than about $100 \mathrm{MeV}$. This is due to the fast energy degradation rate.

This is not the end of the story. When the decay occurs the charged daughter particle carries off a large amount of energy. All of this gets converted to non-thermal photons [36]. In the time it takes for these photons to thermalize, they can find $a^{6} \mathrm{Li}$ (and other nuclei) and photodissociate it if the energy is sufficient to cross the threshold.

We can calculate the non-thermal distribution of the photons following [8, 15, 36]. The initial energy injection is rapidly brought to a quasi-static equilibrium state by pair production off of the background (thermal) photons and inverse Compton scattering. On a longer timescale (but still shorter than the age) processes like photonphoton scattering and Compton scattering redistribute the energy and lead to thermalization. We assume that the thermalization is mainly due to Compton scattering and calculate the differential non-thermal photon number density following [15].

With the expression for the non-thermal photon distribution at hand, one may write the rate for ${ }^{6} \mathrm{Li}$ production as

$$
\begin{aligned}
\frac{d}{d \ln (T)} Y_{6}(T) & =-\frac{n_{B}(T)}{\tau H(T)} f_{L}(T) f_{3} f_{P} f_{b}(T) \\
& +Y_{6}(T) \frac{\Gamma_{6, \gamma}}{H(T)}
\end{aligned}
$$

where subscript "6" stands for ${ }^{6} \mathrm{Li}$ and $\Gamma_{6, \gamma}$ is the total photo-dissociation rate for $\gamma+{ }^{6} \mathrm{Li} \rightarrow$ anything. $Y$ denotes the number density relative to the number density of baryons.

Similarly, the interaction of ${ }^{3} \mathrm{He}$ and $\mathrm{T}$ with the background protons and photons can degrade the nuclear energy. We have checked that these are negligible effects for the temperatures of around $10 \mathrm{keV}$ and lower that we are interested in.

The non-thermal photons may also dissociate the ${ }^{3} \mathrm{He}$ before they can collide with ${ }^{4} \mathrm{He}$ nuclei. To estimate this, we note that the minimum threshold for ${ }^{3} \mathrm{He}$ destruction is about $5.5 \mathrm{MeV}$. In order to produce photons with this energy, one would have to be at a temperature 


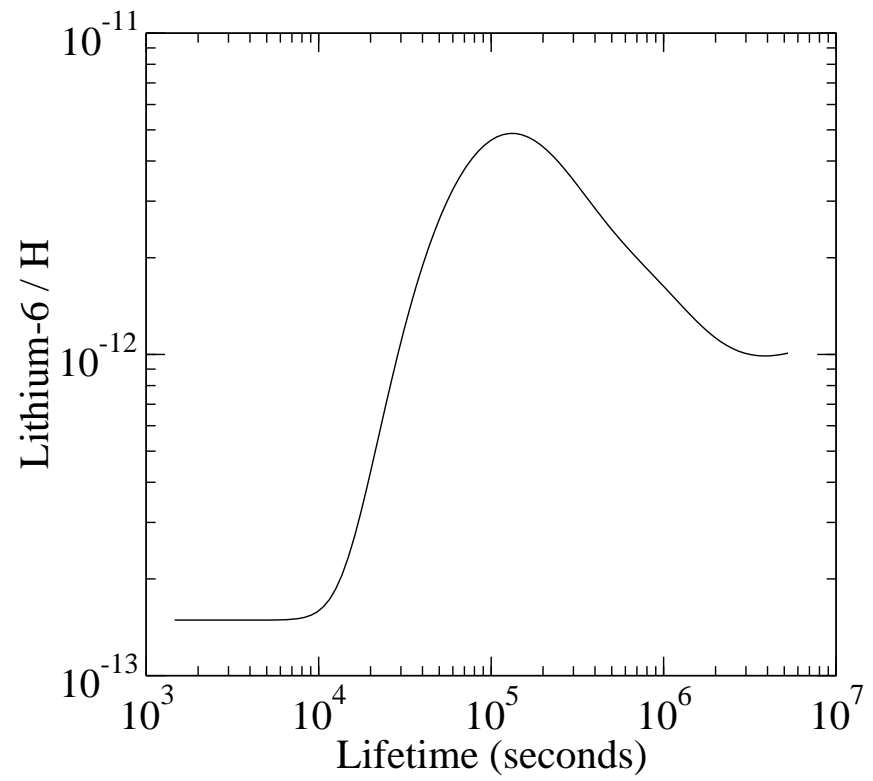

FIG. 2: Curve shows the ratio of number density of ${ }^{6} \mathrm{Li}$ to that of $\mathrm{H}$ produced due to the process described in the text. The model assumed to make this plot is a gravitino SuperWIMP with mass of $100 \mathrm{GeV}$ and a heavier charged slepton like stau. We start with an initial ${ }^{6} \mathrm{Li} / \mathrm{H}$ ratio of $1.4 \times 10^{-13}$.

lower than about $2 \mathrm{keV}$. Thus, this could only be a problem for long lifetimes. Also, the spectrum at these energies is falling sharply [15, 36]. We estimate that this effect cannot be significant unless decay lifetimes are larger than $10^{6}$ seconds and energy released is larger than $\mathrm{TeV}$.

The bottom-line from the above calculation is that it is possible to produce ${ }^{6} \mathrm{Li}$ of order $10^{-12}$ (relative to the number density of $\mathrm{H}$ ) in large regions of decaying charged particle model parameter space. This is clear from the Figure 2 where we have assumed that a slepton is decaying to a gravitino and lepton, with the gravitino having a mass of $100 \mathrm{GeV}$. As the mass of the gravitino is lowered, the amount of ${ }^{6} \mathrm{Li}$ produced decreases. Note that the masses where a reasonable amount of ${ }^{6} \mathrm{Li}$ is produced corresponds to astrophysically interesting lifetimes of about a month [37, 38]. We note in closing that the dissociation by non-thermal photons will be insignificant for models where the mass of the decaying and daughter particles is fine-tuned to be small [6].

\section{PRODUCTION OF LITHIUM-7 AND OTHER REACTIONS}

After the decay, all the ${ }^{4} \mathrm{He}$ nuclei have the orbital kinetic energy $\sim 0.16 \mathrm{MeV}$. The kinetic energy is still not enough to initiate any endothermic nuclear reactions. We therefore consider the following processes ${ }^{3} \mathrm{He}(\alpha, \gamma)^{7} \mathrm{Be}$ and ${ }^{3} \mathrm{H}(\alpha, \gamma){ }^{7} \mathrm{Li}$. Proceeding as in the ${ }^{6} \mathrm{Li}$ production case, we find that this process produces insignificant amounts of ${ }^{7} \mathrm{Li}$ and ${ }^{7} \mathrm{Be}$.

The ${ }^{4} \mathrm{He}$ nuclei could also receive a large kick due to elastic scattering off of the decay produced charged particle. Our calculations show that for small energy releases of order $10 \mathrm{GeV}$, it is possible to produce ${ }^{7} \mathrm{Li}$ in quantities comparable to the standard BBN yield. However, in concrete cases like the Super-WIMP scenario [1, 16], the lifetime for such small energy releases is large which means that the destruction rate due to non-thermal photons is large. The destruction effect dominates and significant amounts of ${ }^{7} \mathrm{Li}$ are not produced.

We also note that the break-up of ${ }^{4} \mathrm{He}$ produces energetic neutrons and protons that in turn can break up the background ${ }^{4} \mathrm{He}$ nuclei. However, we have seen that only about 1 in a million bound ${ }^{4} \mathrm{He}$ is broken up and hence the destruction due to these energetic protons and neutrons is negligible.

\section{DISCUSSION}

We have concentrated here on the collision of ${ }^{3} \mathrm{He}$ with background ${ }^{4} \mathrm{He}$ nuclei that would produce ${ }^{6} \mathrm{Li}$ nuclei. Standard BBN does not produce much ${ }^{6} \mathrm{Li}$ and thus the expectation is that this could be an important probe. The SBBN yield (without decays) is about $10^{-13}$ compared to the number density of H. This should be compared to the measured lithium isotopic ratios in some low metallicity popII stars which (conservatively) range between 0.01 and 0.1. A reasonable constraint on ${ }^{6} \mathrm{Li}$ then is that its abundance should not be much larger than that of ${ }^{7} \mathrm{Li}$ nuclei (about $10^{-10}$ relative to the $\mathrm{H}$ number density). It is of course possible that the ${ }^{6} \mathrm{Li}$ primordial abundance is larger, but then we are left with the question of why ${ }^{6} \mathrm{Li}$ is depleted in much larger amounts than ${ }^{7} \mathrm{Li}$ by astrophysical mechanisms.

We found that in some regions of parameter space it is possible to produce ${ }^{6} \mathrm{Li}$ in quantities that match the observed "plateau" abundance. This does not imply that the decaying charged particle scenario can explain the ${ }^{6} \mathrm{Li}$ abundance. A full calculation including all the light elements would be necessary to ascertain that. We also note that there are astrophysical scenarios wherein the observed abundance of ${ }^{6} \mathrm{Li}$ finds an explanation (e.g., 39, 40, 41, 42, 43, 44, 45]).

The processes we have described here are clearly relevant for determining the light element abundances. Much more work needs to be done before these processes can be combined with previous analyses to put bounds on the model parameter space. Throughout this work we have neglected the bound states of other light elements. The fraction of bound states of $\mathrm{D}, \mathrm{T},{ }^{3} \mathrm{He},{ }^{6} \mathrm{Li}$ and ${ }^{7} \mathrm{Li}$ are small owing to the much smaller abundance of the respective light elements. However, they could still have significant effects because of enhanced nuclear cross-sections. The correct treatment of this problem including all the 
cross-sections and bound states is beyond the scope of present work.

In summary, we have studied an aspect of the decay of charged particles in the early universe. We looked at the electromagnetic bound states of the charged particle with ${ }^{4} \mathrm{He}$ nuclei and considered the effects of the decay on the light element abundances. We found that cosmologically relevant quantities of ${ }^{6} \mathrm{Li}$ could be produced as a result of these decays.

\section{ACKNOWLEDGEMENTS}

MK would like to acknowledge NSF grant PHY0555689. AR would like to acknowledge NSF grant PHY0354993.

[1] J. L. Feng, A. Rajaraman, and F. Takayama, Phys. Rev. Lett. 91, 011302 (2003).

[2] J. L. Feng, A. Rajaraman, and F. Takayama, Phys. Rev. D68, 085018 (2003).

[3] L. Covi, H.-B. Kim, J. E. Kim, and L. Roszkowski, JHEP 05, 033 (2001).

[4] L. Covi, L. Roszkowski, R. Ruiz de Austri, and M. Small, JHEP 06, 003 (2004).

[5] A. Brandenburg et al., Phys. Lett. B617, 99 (2005).

[6] K. Sigurdson and M. Kamionkowski, Phys. Rev. Lett. 92, 171302 (2004).

[7] S. Profumo, K. Sigurdson, P. Ullio, and M. Kamionkowski, Phys. Rev. D71, 023518 (2005).

[8] S. Dimopoulos, R. Esmailzadeh, L. J. Hall, and G. D. Starkman, Nucl. Phys. B311, 699 (1989).

[9] M. Kawasaki and T. Moroi, Prog. Theor. Phys. 93, 879 (1995).

[10] M. Y. Khlopov, Y. L. Levitan, E. V. Sedelnikov, and I. M. Sobol, Phys. Atom. Nucl. 57, 1393 (1994).

[11] E. V. Sedelnikov, S. S. Filippov, and M. Y. Khlopov, Phys. Atom. Nucl. 58, 235 (1995).

[12] E. Holtmann, M. Kawasaki, and T. Moroi, Phys. Rev. Lett. 77, 3712 (1996).

[13] E. Holtmann, M. Kawasaki, K. Kohri, and T. Moroi, Phys. Rev. D60, 023506 (1999).

[14] K. Jedamzik, Phys. Rev. Lett. 84, 3248 (2000).

[15] R. H. Cyburt, J. R. Ellis, B. D. Fields, and K. A. Olive, Phys. Rev. D67, 103521 (2003).
[16] J. L. Feng, A. Rajaraman, and F. Takayama, Phys. Rev. D68, 063504 (2003).

[17] K. Jedamzik, Phys. Rev. D70, 063524 (2004).

[18] M. Kawasaki, K. Kohri, and T. Moroi, Phys. Rev. D71, $083502(2005)$.

[19] F. D. Steffen, JCAP 0609, 001 (2006).

[20] K. Jedamzik, K.-Y. Choi, L. Roszkowski, and R. Ruiz de Austri, JCAP 0607, 007 (2006).

[21] K. Jedamzik, arXiv:hep-ph/0604251 (2006).

[22] V. V. Smith, D. L. Lambert, and P. E. Nissen, Astrophys. J. 408, 262 (1993).

[23] V. V. Smith, D. L. Lambert, and P. E. Nissen, Astrophys. J. 506, 405 (1998).

[24] P. E. Nissen, D. L. Lambert, F. Primas, and V. V. Smith, Astron. \& Astrophys.348, 211 (1999).

[25] E. Vangioni-Flam et al., New Astronomy 4, 245 (1999).

[26] P. E. Nissen, M. Asplund, V. Hill, and S. D'Odorico, Astron. \& Astrophys.357, L49 (2000).

[27] M. Asplund et al., Astrophys. J. 644, 229 (2006).

[28] R. N. Cahn and S. L. Glashow, Science 213, 607 (1981).

[29] K. M. Belotsky et al., Grav. Cosmol. 11, 16 (2005).

[30] D. Fargion and M. Khlopov, arXiv:hep-ph/0507087 (2005).

[31] M. Y. Khlopov, Pisma Zh. Eksp. Teor. Fiz. 83, 3 (2006).

[32] M. Pospelov, arXiv:hep-ph/0605215 (2006).

[33] K. Kohri and F. Takayama, arXiv:hep-ph/0605243 (2006).

[34] M. Arneodo et al., Nucl. Phys. B483, 3 (1997).

[35] R. J. Protheroe, T. Stanev, and V. S. Berezinsky, Phys. Rev. D51, 4134 (1995).

[36] M. Kawasaki and T. Moroi, Astrophys. J. 452, 506 (1995).

[37] M. Kaplinghat, Phys. Rev. D72, 063510 (2005).

[38] J. A. R. Cembranos, J. L. Feng, A. Rajaraman, and F. Takayama, Phys. Rev. Lett. 95, 181301 (2005).

[39] D. L. Lambert, in AIP Conf. Proc. 743: The New Cosmology: Conference on Strings and Cosmology, edited by R. E. Allen, D. V. Nanopoulos, and C. N. Pope (PUBLISHER, ADDRESS, 2004), pp. 206-223.

[40] E. Rollinde, E. Vangioni, and K. Olive, Astrophys. J. 627, 666 (2005).

[41] H. Reeves, in EAS Publications Series, edited by G. Alecian, O. Richard, and S. Vauclair (PUBLISHER, ADDRESS, 2005), pp. 15-19.

[42] B. B. Nath, P. Madau, and J. Silk, Mon.Not.Roy.As.Soc.366, L35 (2006).

[43] T. K. Suzuki and S. Inoue, Astrophys. J. 573, 168 (2002).

[44] N. Prantzos, Astron. \& Astrophys.448, 665 (2006).

[45] T. Prodanović and B. D. Fields, Astrophys. J. Lett.645, L125 (2006). 